\title{
Microsphere-based Optical System for Biosensor Applications
}

\author{
Ali Serpengüzel, ${ }^{*}$ Senol Isçi, ${ }^{\dagger}$ Temel Bilici, ${ }^{\dagger}$ and Adnan Kurt \\ Koç University, Microphotonics Research Laboratory, Physics Department, \\ Rumeli Feneri Yolu, Sariyer, Istanbul 34450 Turkey
}

\begin{abstract}
Optical microsphere resonators have been recently utilized in quantum optics, laser science, spectroscopy, and optoelectronics and attracted increasing interest due to their unique optical properties. Microspheres possess high quality factor (Q-factor) optical morphology dependent resonances, and have relatively small volumes. High-Q morphology dependent resonances are very sensitive to the refractive index change and microsphere uniformity. These tiny optical cavities, whose diameters may vary from a few to several hundred micrometers, have resonances with reported Q-factors as large as $3 \times 10^{9}$. Due to their sensitivity, morphology dependent resonances of microspheres are also considered for biosensor applications. Binding of a protein or other biomolecules can be monitored by observing the wavelength shift of morphology dependent resonances. A biosensor, based on this optical phenomenon, can even detect a single molecule, depending on the quality of the system design. In this work, elastic scattering spectra from the microspheres of different materials are experimentally obtained and morphology dependent resonances are observed. Preliminary results of unspecific binding of biomolecules onto the microspheres are presented. Furthermore, the morphology dependent resonances of the microspheres for biosensor applications are analyzed theoretically both for proteins such as bovine serum albumin.
\end{abstract}

Keywords: Dielectric microsphere, evanescent coupling, integrated optoelectronics, microsphere resonator, morphology dependent resonances, optical biosensor, optical coupler, optical fiber, optical resonance, whispering gallery modes.

\section{INTRODUCTION}

Dielectric microspheres have found various photonic applications ${ }^{1}$. Morphology dependent resonances (MDR's) or alternatively whispering gallery modes of dielectric microspheres provide the necessary optical feedback for applications in spectroscopy, laser science, and optical communications. Among these applications are novel microlasers, optical couplers, and optical filters. ${ }^{2}$ Low threshold lasing from Nd-doped silica microspheres ${ }^{2}$, polymer microsphere lasers ${ }^{3}$ and Raman lasers have been demonstrated.

Due to their sensitivity, MDR's of microspheres are also considered for biosensor applications. Binding of a protein or other biomolecules can be monitored by observing the wavelength shift of MDR's. A biosensor, based on this optical phenomenon, can even detect a single molecule, depending on the quality of the system design. In this work, elastic scattering spectra from the microspheres of different materials are experimentally obtained and MDR's are observed. Preliminary results of unspecific binding of biomolecules onto the microspheres are presented. Furthermore, the wavelength shift of the MDR's for biosensor applications are analyzed theoretically for proteins such as bovine serum albumin (BSA). Elastically scattered light is detected from a microsphere placed on an optical fiber half coupler (OFHC). ${ }^{4}$ The MDR's in the elastic scattering spectrum and associated dips in the transmission spectrum are experimentally observed. This coupling geometry is suitable for the manufacturing of optoelectronic devices for biosensor applications. ${ }^{5}$

\footnotetext{
* Correspondence should be addressed to Ali Serpengüzel, aserpenguzel@ku.edu.tr; phone: + 90 (212) 338-1312; fax: + 90 (212) 338-1547, http://home.ku.edu.tr/ aserpenguzel, http://microphotonics.ku.edu.tr, Koç University, Microphotonics Research Laboratory, Physics Department, Rumeli Feneri Yolu, Sariyer, Istanbul 34450 Turkey. The authors are with Koç University, Microphotonics Research Laboratory, Department of Physics, Rumeli Feneri Yolu, Sariyer, Istanbul 34450 Turkey. † Senol Isçi is currently at the Scientific and Technical Research Council of Turkey (TUBITAK), National Institute of Electronics and Cryptology (UEKAE), P.K. 74, Gebze, Kocaeli 41470 Turkey. \$ Temel Bilici is currently at Boston University, Department of Electrical and Computer Engineering, 8 St. Mary's Street, Boston, Massachusetts 02215 USA.
} 


\section{MORPHOLOGY DEPENDENT RESONANCES}

The MDR's of the microsphere can be understood using geometrical optics. As the light propagates around the microsphere, it is confined by almost total internal reflection (TIR). ${ }^{6}$ After circumnavigating the microsphere, the light wave returns to the original starting point in phase to interfere constructively with itself. This constructive interference can occur only at certain discrete MDR wavelengths. ${ }^{7}$ Each MDR is characterized by a mode number (n) and a mode order (l). Physically, (n) indicates the number of nodes in the internal intensity distribution as the polar angle is varied from $0^{\circ}$ to $180^{\circ}$. The mode order (l) indicates the number of nodes in the internal intensity distribution in the radial direction. For each set of mode number (n) and mode order (l), there is a transverse electric (TE) and transverse magnetic (TM) MDR. For a given microsphere, the MDR occurs at specific value of the size parameter, $\mathrm{x}_{\mathrm{n}, 1}$, which is given by $2 \pi \mathrm{a} / \lambda_{\mathrm{n}, 1}$, where $\lambda_{\mathrm{n}, 1}$ is the light wavelength in vacuum and a is the radius of the microsphere. These MDR's have been verified experimentally at optical wavelengths with micrometer-sized spheres. The greatest impediment to the use of microsphere resonators in practical devices has been the difficulty of efficiently light coupling into and out of the spheres. To couple light into or out of the microsphere, it is necessary to overlap the evanescent field of the MDR with the evanescent field of the TIR. Such coupling has been implemented, by the use of thin tapered optical fibers, planar waveguide couplers, side-polished optical fibers, or high-index-prisms. If a microsphere is placed near the angled surface, and within the evanescent field of the fiber-optic core, then there is an efficient energy exchange in resonance between the waveguide mode of the fiber and the MDR of the microsphere. ${ }^{8}$

\section{EXPERIMENTAL SETUP}

A common approach is to polish down the cladding of an optical fiber to the point, where the evanescent field is locally exposed. ${ }^{9}$ In the experiment, the OFHC is fabricated from a $810 \mathrm{~nm}$ single mode fiber with a core radius of $1.9 \mu \mathrm{m}$ and a refractive index of 1.462, and a cladding radius of $62.5 \mu \mathrm{m}$ with refractive index of 1.457 , which is laid on a glass substrate with a low curvature. The cladding of the fiber below the microsphere is shaved down to $0.7 \mu \mathrm{m}$ in order to approach the core of the fiber. This excitation geometry effectively becomes the optical equivalent of a Gaussian beam with an infinite skirt length passing near the microsphere. The transmission loss from the OFHC corresponds to $0.8 \%$.

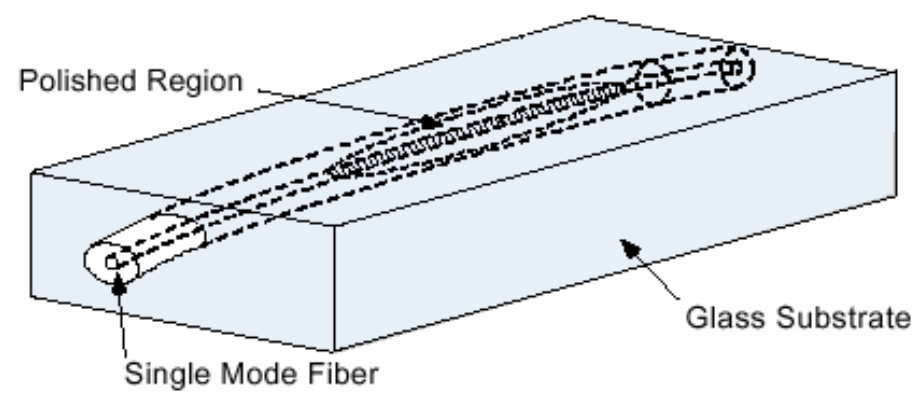

Figure 1. The schematic of the optical fiber half coupler.

A schematic of the optical fiber half coupler (OFHC) is shown in Fig. 1. The microsphere used in the experiment is a BK7 type glass with a radius $\mathrm{a}=500 \mu \mathrm{m}$ and a refractive index of 1.502 . MDR's of the microsphere are excited by using a tunable distributed feedback (DFB) semiconductor laser. The schematic of the experimental setup is given in Fig. 2. Wavelength tuning is achieved by tuning the temperature of the DFB with a laser diode controller. The elastically scattered polarization-independent light at $90^{\circ}$ is detected by using an unamplified windowless wide area (4 mm x $4 \mathrm{~mm}$ ) silicon photodiode placed approximately $2 \mathrm{~mm}$ away from the microsphere-OFHC system as seen in Fig. 2. The photodetector signal is sent to a digital oscilloscope for monitoring and data acquisition. The optical power and the wavelength of the transmitted light are measured by an optical multimeter (OMM) with a silicon power/wave head. Device controls and data acquisition are performed with the IEEE-488 standard GPIB interface. 


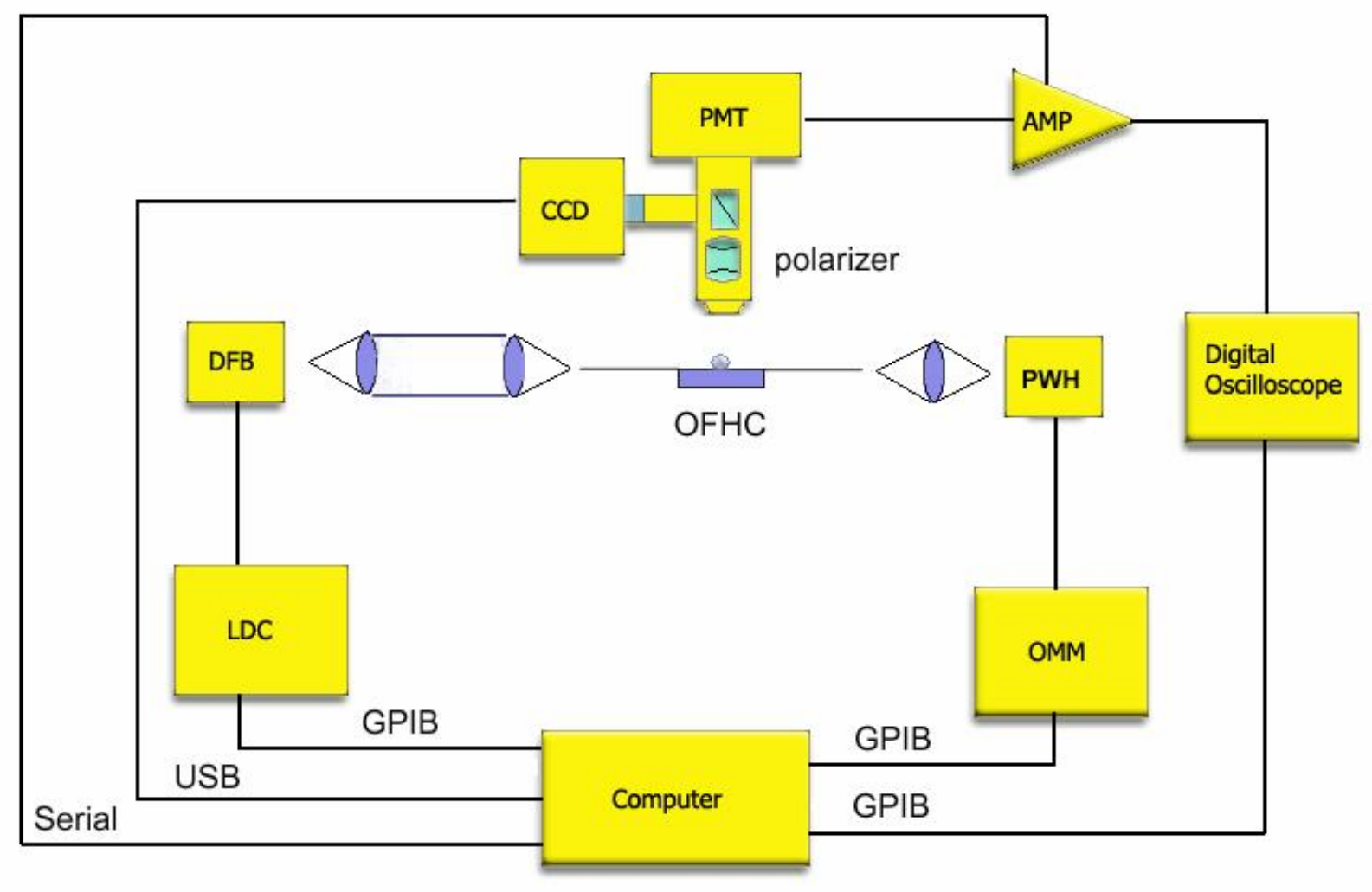

Figure 2. The schematic of the experimental setup.

\section{ELASTIC LIGHT SCATTERING}

Fig. 3 shows the elastic scattering and the power transmission spectra. MDR's in the elastic scattering and the associated dips in the transmitted power spectra are clearly observed. The temperature of the DFB laser is tuned between $19{ }^{\circ} \mathrm{C}$ and $30{ }^{\circ} \mathrm{C}$ at a constant current of $31.2 \mathrm{~mA}$. This temperature range corresponds to a wavelength range from $811.83 \mathrm{~nm}$ to $812.32 \mathrm{~nm}$. At the MDR wavelengths, the laser is coupled out of the fiber into the microsphere, which results in dips in the transmitted signal. It is important to note that the fractional depth of the dips in the transmission spectra is not the same for all MDR's. This is due to different Q-factors and coupling efficiencies of the MDR's.

The scattered signal intensity increases with the decreasing the distance of the photodiode to the microsphere. There is a background due to the OFHC surface imperfections. ${ }^{10}$ With a larger refractive index or smaller size microsphere, the MDR peaks would be more clearly observable in the spectrum. The bandwidths of the MDR's are measured to be 0.05 $\mathrm{nm}$ and the separation between the same mode order (l) MDR's with consecutive mode numbers ( $\mathrm{n}$ ), $\Delta \lambda$, is measured to be about $0.14 \mathrm{~nm}$, which is consistent with the calculation based on the Mie scattering theory given by $\Delta \lambda=$ $\left(\lambda^{2} \arctan \left(\mathrm{m}^{2}-1\right)^{1 / 2}\right) /\left(2 \pi \mathrm{a}\left(\mathrm{m}^{2}-1\right)^{1 / 2}\right){ }^{11}$

The measured quality (Q) factor of the MDR's in the polarization-independent scattering spectrum is approximately of $10^{4}$. The high Q factor MDR's are superimposed upon a background of low Q-factor MDR's. Since no polarizer is used in the experiment, the spectrum gets contributions from both the TE and the TM modes, resulting in spectrally merged MDR's. 

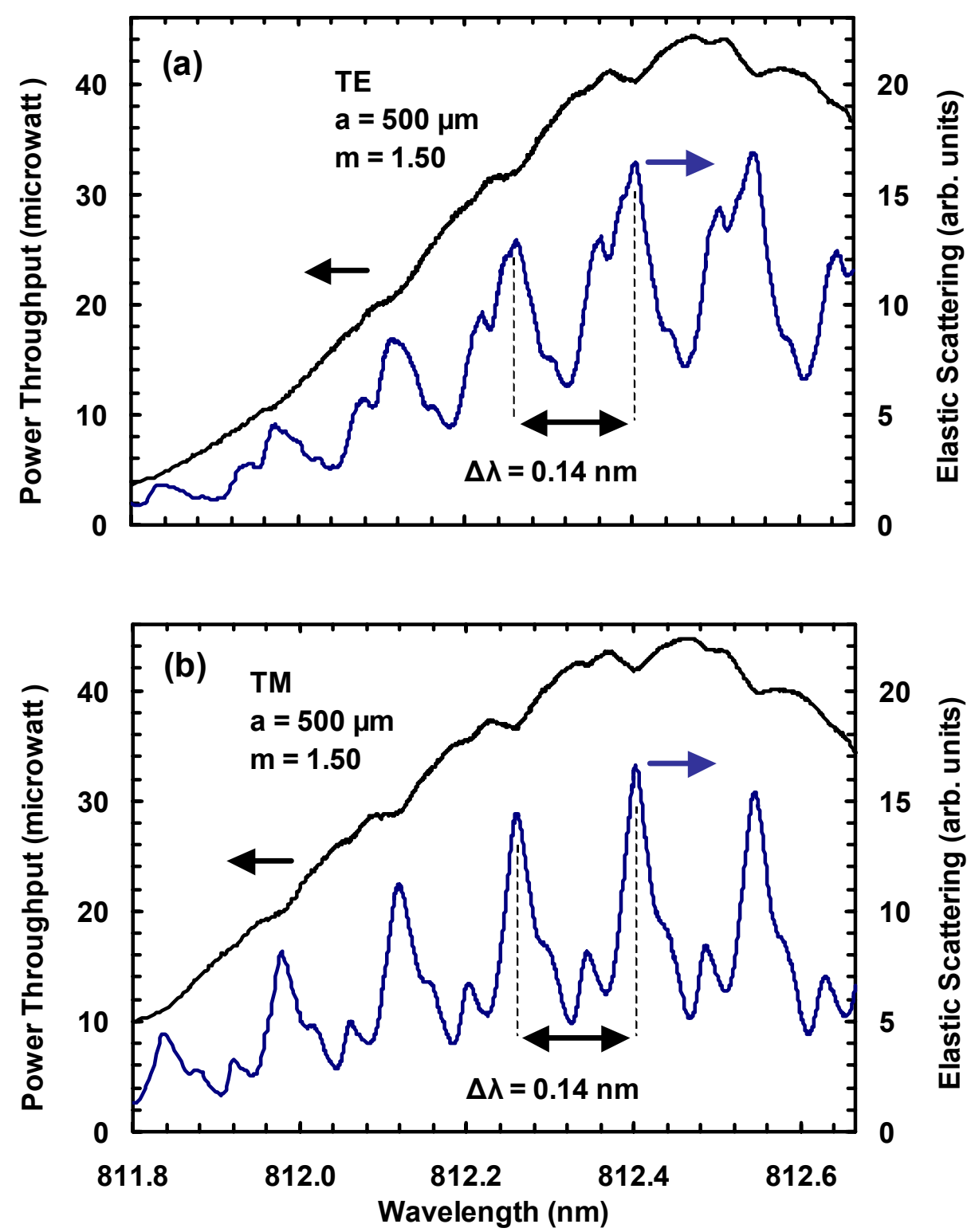

Figure 3. The (a) TE and (b) TM polarized elastic scattering intensity and the power transmission spectra from the glass microsphere.

\section{BIOSENSOR APPLICATIONS}

By measuring the shift of the MDR location, it might be possible to detect the binding of the molecules to the surface of the microsphere. Figure 4 depicts the schematic of the proposed method with the original microsphere (a) spectrum and (b) the final shifted microsphere spectrum with the attached biomolecules. 

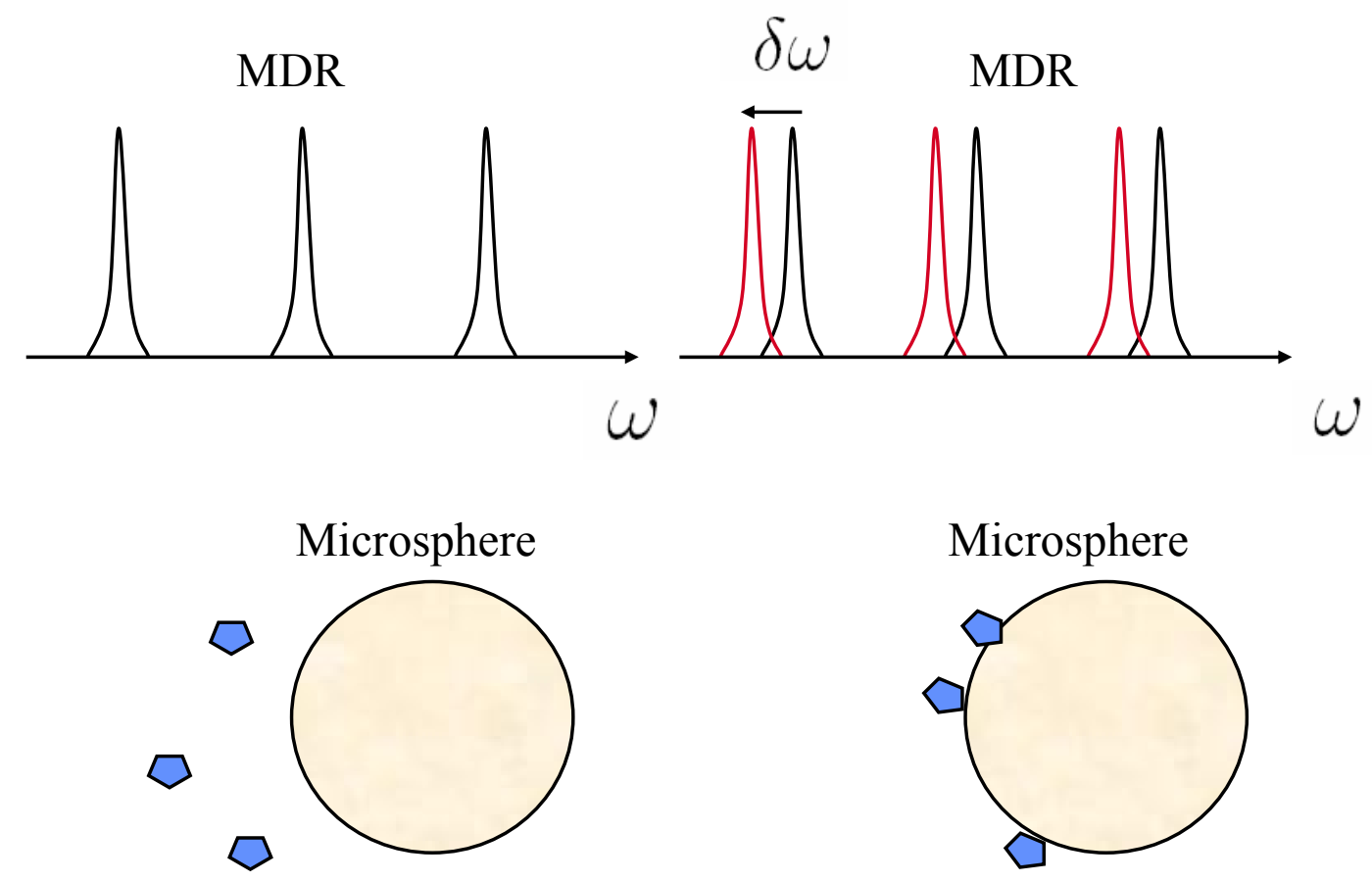

biomolecules

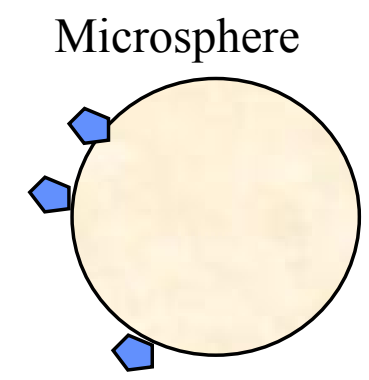

biomolecules

Figure 4. The schematic of the proposed method with the original microsphere (a) spectrum and (b) the final shifted microsphere spectrum with the attached biomolecules.

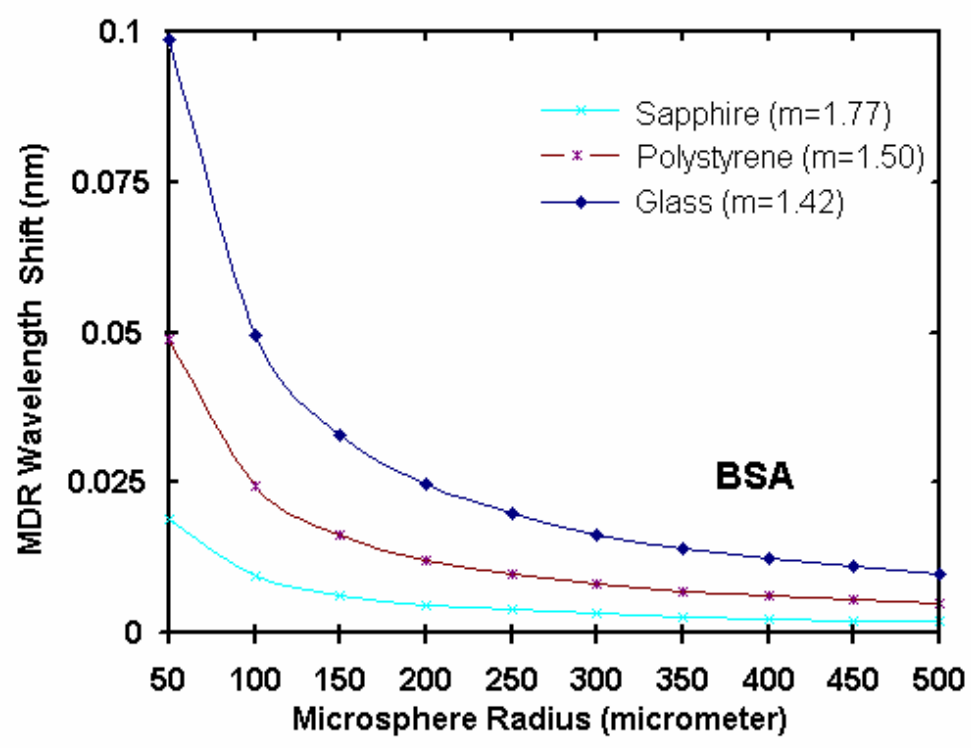

Figure 5. The MDR wavelength shift as a function of the microsphere radius at an excitation wavelength of 1550 $\mathrm{nm}$ for bovine serum albumin (BSA) molecule assuming a full monolayer coating of the microsphere surface. 
In Fig. 5, we have calculated the MDR wavelength shift as a function of the microsphere radius at an excitation wavelength of $1550 \mathrm{~nm}$ for bovine serum albumin (BSA) molecule assuming a full monolayer coating of the microsphere surface. ${ }^{12}$ The MDR wavelength shifts on the order of $0.01-0.1 \mathrm{~nm}$ are well within our detection range.

\section{CONCLUSIONS}

We have demonstrated the excitation of MDR's of glass microspheres using an optical fiber half coupler (OFHC) and, a temperature tunable distributed feedback (DFB) laser. MDR peaks in the elastic scattering spectra and associated dips in the transmission spectra are observed experimentally. We have also calculated the relative shifts in the MDR wavelengths using a full monolayer coating of the microsphere surface with the bovine serum albumin molecule. The MDR wavelength shifts on the order of 0.01-0.1 nm are well within our detection range. The OFHC, microsphere, and photodetector system shows potential as a biomolecular sensor.

\section{ACKNOWLEDGMENTS}

We would like to acknowledge the partial support of this research by the Scientific and Technical Research Council of Turkey (TUBITAK) Grant No: TBAG-1952 and the European Office of Aerospace Research and Development (EOARD) Grants No: F61775-01-WE062 and FA8655-02-M-4086.

\section{REFERENCES}

1. S. M. Spillane, T. J. Kippenberg, and K. J. Vahala, "Ultralow-threshold Raman laser using a spherical dielectric microcavity", Nature, vol. 415, pp. 621-623, Feb. 2002.

2. V. Lefèvre-Seguin and S. Haroche, "Towards cavity-QED experiments with silica microspheres", Mat. Sci. Eng., vol. B48 pp. 53-58, 1997.

3. M. Kuwata-Gonokami and K. Takeda, "Polymer whispering gallery mode lasers", Opt. Mat., vol. 9, pp. 12-17, Jan. 1998.

4. T. Bilici, S. Isçi, A. Kurt, and A. Serpengüzel, "Microsphere Based Channel Dropping Filter with Integrated Photodetector," IEEE Photon. Technol. Lett., 16, no. 2. (2004) (to be published).

5. S. Isçi, T. Bilici, A. Kurt, and A. Serpengüzel, "Morphology Dependent Resonances of Optical Microsphere Resonators for the Realization of Passive WDM Components," Opt. Eng., 43, (2004) (to be published).

6. M. Pelton and Y. Yamamoto, "Ultralow threshold laser using a single quantum dot and a microsphere cavity", Phys. Rev. A, vol. 59, no. 3, pp. 2418-2421, March 1999.

7. B. R. Johnson, "Morphology-dependent resonances of a dielectric sphere on a conducting plane", J. Opt. Soc. Am. A, vol. 11, no. 7, pp. 2055-2064, July 1994.

8. J.-P. Laine, B. E. Little, and H. A. Haus, "Etch-Eroded Fiber coupler for whispering-gallery-mode excitation in high Q silica microspheres”, IEEE Photon. Technol. Lett., vol. 11, no. 11, pp. 1429-1430, Nov. 1999.

9. O. Parrriaux, S. Gidon, and A. A. Kuznetsov, "Distributed coupling on polished single-mode optical fibers", Appl. Opt., vol. 20, no.14, pp. 2420-2423, July 1981.

10. A. Serpengüzel, S. Arnold, and G. Griffel, "Photonic atoms: Enhanced light coupling", in "Microcavities and photonic bandgaps: Physics and applications", J. Rarity and C. Weisbuch, Kluwer Academic Publishers, Netherlands, 1995.

11. R. Jia, D. Jiang, P. Tan, B. Sun, J. Zhang, and Y. Lin, "Photoluminescence study of CdSexS1-x quantum dots in a glass spherical microcavity", Chin. Phys. Lett., vol. 18, no. 10, pp. 1350-1352, 2001.

12. S. Arnold, M. Khoshima, I. Teraoka, S. Holler, and F. Vollmer, "Shift of whispering gallery modes in microspheres by protein adsorption," Opt. Lett. 28, no. 4, pp. 272-274, 2003. 